Article

\title{
High Fidelity Multi-Objective Design Optimization of a Downscaled Cusped Field Thruster
}

\author{
Thomas Fahey ${ }^{1,+}$, Angus Muffatti ${ }^{2, \dagger}$ and Hideaki Ogawa ${ }^{1, *}$ \\ 1 School of Engineering, RMIT University, Melbourne, Victoria 3001, Australia; s3163948@student.rmit.edu.au \\ 2 Aerospace Systems Pty Ltd., Prahran, Victoria 3181, Australia; angus@aerospacesystems.com.au \\ * Correspondence: hideaki.ogawa@rmit.edu.au; Tel.: +61-3-9925-6042 \\ $\dagger$ These authors contributed equally to this work.
}

Received: 27 September 2017; Accepted: 11 November 2017; Published: 18 November 2017

\begin{abstract}
The Cusped Field Thruster (CFT) concept has demonstrated significantly improved performance over the Hall Effect Thruster and the Gridded Ion Thruster; however, little is understood about the complexities of the interactions and interdependencies of the geometrical, magnetic and ion beam properties of the thruster. This study applies an advanced design methodology combining a modified power distribution calculation and evolutionary algorithms assisted by surrogate modeling to a multi-objective design optimization for the performance optimization and characterization of the CFT. Optimization is performed for maximization of performance defined by five design parameters (i.e., anode voltage, anode current, mass flow rate, and magnet radii), simultaneously aiming to maximize three objectives; that is, thrust, efficiency and specific impulse. Statistical methods based on global sensitivity analysis are employed to assess the optimization results in conjunction with surrogate models to identify key design factors with respect to the three design objectives and additional performance measures. The research indicates that the anode current and the Outer Magnet Radius have the greatest effect on the performance parameters. An optimal value for the anode current is determined, and a trend towards maximizing anode potential and mass flow rate is observed.
\end{abstract}

Keywords: electric propulsion; cusped field thruster; multi-objective design optimization

\section{Introduction}

The implementation of electric propulsion (EP) thrusters on spacecraft is largely driven by weight reductions over traditional chemical propulsion thrusters. EP has a distinct payload reduction advantage over chemical propulsion and offers increased operational life, in excess of 10,000 h, and high specific impulse $I_{s p}$, of $1500-4000 \mathrm{~s}$, but relatively low thrust values of around $30-230 \mathrm{mN}$ [1], which is useful for long range missions and small attitude adjustments for satellites [2]. EP offers improved specific impulse and operational lifetimes over chemical thrusters with a significant reduction in the amount of propellant required. The reduction in propellant due to reduced fuel consumption and low system mass leads to a significant reduction in launch costs, making this technology desirable. Keller et al. [3] has identified several upcoming satellite missions that require smaller EP thrusters for increased precision attitude and control.

The gridded ion thruster (GIT) and the Hall effect thruster (HET) are high efficiency flight tested examples of EP. Both of these propulsion concepts are well understood and have values of $I_{s p}$ ranges from 1500 to $6000 \mathrm{~s}$ [4]. Both of these designs provide great performance but also come with drawbacks such as system complexity and thruster erosion from impinging ions on grids or channel walls [5], which limits their operational lifetimes. First developed by Thales Electron Devices in 1999 as the Highly Efficient Multi-stage Plasma Thruster (HEMP-T) [3], based on travelling wave 
tube technology, the Cusped Field Thruster (CFT) concept has a simplified design and demonstrates improved performance characteristics over GIT and HET, with $I_{s p}$ in the range of 2000-3000 s, and an estimated minimum operational lifetime of over 16,000 $\mathrm{h}$ [6]. Since the first HEMP-T patent by Thales Electron Devices (TED) in 1999, similar thruster designs have been developed and tested by different institutions around the world; MIT's Diverging Cusped-Field Thruster (DCFT) [7], Stanford's DCFT [8] and the Harbin Institute of Technology's CFT [9].

The CFT shares the HET axial electric field and radial magnetic field, using the Hall Current to ionize the propellant. The CFT uses Permanent Periodic Magnets (PPM) in series, aligned axially along the chamber with reversing polarity to contain the plasma through the magnetic mirror effect, and avoids plasma contact with the chamber walls that typically results in wall erosion. Wall erosion is one of the main impediments on HET and GIT operational lifetimes and experimental testing on CFT designs has shown no erosion [5]. The arrangement of the PPM reduces charge losses by restricting the radial motion of the plasma, resulting in high ion beam efficiencies similar to GIT, in the region of $80-90 \%$, compared to $60-70 \%$ for HET [5]. Between the magnetic stages of the PPM, a cusp region forms where the magnetic field runs radially from the chamber wall, repelling the plasma [5]. This impedes the electrons from moving upstream by the magnetic mirror effect. The magnetic field strength is optimized to concentrate the ion beam discharge to the center of the engine. The cusp regions effectively divide the engine in to separate magnetic cell stages.

The magnetic field strength $B$ is chosen such that the Larmor radius of the electrons is much smaller than the inner geometry of the discharge chamber. This effectively confines the electrons to the center of the engine that in turn helps to electrostatically confine the ions, of which the magnetic field has little effect upon as the mean free path of the ion is significantly smaller than its gyro radius and is not considered magnetized [10]. Ions that escape this confinement contribute to an overall positive charge of the chamber wall and therefore an enhancement of ion beam formation [11]. The CFT has the ionization and acceleration zone separation characterized by the GIT. The thruster also features a steep potential drop occurring immediately after the exit cusp whilst the plasma potential is maintained at a relatively constant level throughout the engine [5,11-13]. This is mainly due to the magnetic cusp at the thruster exit. The electrons emitted from the cathode are trapped here and form the cloud. The neutralizer, while not necessary for performance, also prevents the discharge from interfering with other spacecraft systems. The confined electron cloud acts as a virtual acceleration grid, like the GIT, resulting in high efficiencies and ions close to the potential of the anode.

Focusing on the need for micro thrust propulsion solutions for high precision station keeping of satellites, a parametric study to downscale the original HEMP-T concept was conducted by Keller et al. [3]. Reducing the size of the CFT could result in better performance over current EP thrusters, with reduced weight, reduced fuel consumption and therefore more launch cost savings for satellites applications. Keller et al. [3] conducted a performance parameterization study of the HEMP-T design. In their efforts to scale down the size of the thruster into the micro-newton thrust range, they have shown the relation of several inter-related sizing parameters. Testing various thruster configurations and the sensitivity of parameters (chamber diameter, magnetic ring outer diameter, the distance between magnetic stages and the number of stages) to four output performance measures (mass flow, anode current, plume divergence, acceleration efficiency), an approximate range of values for the micro-newton thrust level was obtained. These specifications provide some insight into the sizing sensitivity of the design and deliver key indicators in the way of thruster sizing ratios such as increasing magnetic field intensity for decreasing discharge chamber diameters to reduce losses. However, the performance remained considerably low in comparison, with the larger scale counterparts with a maximum $I_{s p}$ achieved of $2000 \mathrm{~s}$, whereas the higher end of the performance ranged upwards of $3000 \mathrm{~s}$ [6]. These results reflect the geometric scaling challenges as explored with closed drift thrusters [14].

A state-of-the-art methodology to investigate such higher end performance ranges has been developed by coupling an analytical global-variable based steady-state power and plasma model 
solver and a multi-objective design optimization (MDO) capability based on evolutionary algorithms. This approach enables robust and effective population-based optimization, assisted by surrogate modeling, which can reduce the computational cost by approximating the model calculation in lieu of expensive true computational evaluation. The coupled simulation/MDO approach builds on the preceding work by Muffatti and Ogawa [15] through the utilization of a higher fidelity simulation for more accurate results. This paper presents the outcomes of a multi-objective design optimization of a small-scale CFT analysis that has been conducted for three major objectives commonly targeted in spacecraft propulsion; that is, (1) thrust $T$, (2) efficiency $\eta_{t}$, and (3) specific impulse $I_{s p}$. The magnetic topology is examined for representative high-performance configurations to investigate the underlying thruster physics. The surrogate models are trained with the archive of solutions evaluated during the optimization. They are used to perform variance-based global sensitivity analysis to identify the key design factors as well as an additional large-scale optimization solely employing the surrogate prediction to better capture the effects of the design parameters on the CFT's performance.

\section{Materials and Methods}

The key indicators of thruster performance, thrust $T$, total efficiency $\eta_{t}$, and specific impulse $I_{s p}$, are used to determine the performance of the downscaled CFT in comparison to similar propulsion devices. Starting with the basic relations of spacecraft propulsion thrust $T$ equating with force on a charged particle in an electric field, specific impulse $I_{s p}$ and power $P_{a}[2,10,16]$.

$$
\begin{gathered}
T=v \cdot m_{f}=e \cdot E \\
I_{s p}=\frac{T}{m_{f} g} \\
P=\frac{1}{2} m_{f} v^{2}=\Phi_{a} I_{a}
\end{gathered}
$$

where $E$ is the magnitude of the electric field, $\Phi_{a}$ and $I_{a}$ are the anode voltage and current, respectively, and $m$ is the mass flow rate of propellant (anode and cathode). The total efficiency is defined by the ratio of jet power to anode power $P_{a}$. However, for HET, anode efficiency is generally utilized, defined as:

$$
\eta_{a}=\frac{T^{2}}{2 m_{a} P_{a}}
$$

Expansion on the component efficiencies introduces more measurable voltage $\eta_{v}$, beam $\eta_{b}$, and utilization efficiencies $\eta_{u}$ [17]. Voltage efficiency refers to how efficiently the ions are accelerated by the anode potential; the beam efficiency relates the anode current to the beam current and the utilization efficiency refers to how much propellant is ionized to create the ion beam.

$$
\begin{gathered}
\eta_{a}=\frac{1}{Q^{2}}\left(\frac{\frac{1}{2} m_{i} \overline{v_{i r}^{2}}}{e V_{a}}\right)\left(\frac{I_{b}}{I_{a}}\right)\left(\frac{I_{b}}{e \dot{m} / m_{i}}\right) \cos ^{2} \theta \\
=\frac{1}{Q^{2}} \eta_{v} \eta_{b} \eta_{u} \cos ^{2} \theta
\end{gathered}
$$

From Goebel and Katz [2], the beam current can be determined through the mass flow rate.

$$
I_{b}=e \cdot m_{a} / M
$$

where the subscript $b$ indicates the ion beam, $Q$ is the averaged ionic charge, $I_{b}$ is the total ion current in the beam, $\cos ^{2} \theta$ accounts for plume divergence where ions are not accelerated parallel to the engine axis, $M$ is the mass of propellant atoms $\left(2.18 \times 10^{-25} \mathrm{~kg}\right.$ for $\left.\mathrm{Xe}\right)$ and $e$ is the elementary charge $\left(1.602 \times 10^{-19} \mathrm{C}\right)$. The propellant is assumed to be Xenon, which is typical for CFT. Equation (6) is a 
basic equation that assumes 100\% ionization and singly ionized for HETs, while it would not fully describe low ionization at low voltages and multiply charged ions at high voltages, which could occur in practical applications.

A key feature of the CFT concept is the high voltage operational ranges, typically between 500 and $2000 \mathrm{~V}$. Keller et al. [3] found anode voltages below $500 \mathrm{~V}$ resulted in lower efficiencies at the same mass flow rates, which was supported by the results of Ma et al. [9]. Thus, the effects of low ionization were not captured by this study. The experimental study and theoretical characterization of thrust in Keller, et al. [3] assume that the ion current can be approximated by the anode current with a less than $20 \%$ error and that the particles are singly ionized totally. Matlock [17] measures the ion beam current for the DCFT through electrostatic probing. The mass utilization efficiency, described in part in Equation (7), is sensitive to the beam current and mass flow. As a result, this study assumes that the ion beam current can be sufficiently approximated by Equation (6), with the consideration of presence of up to $20 \%$ doubly charged ions in the mass utilization efficiency for the MDO process. This is detailed in Goebel and Katz [2], where the mass utilization efficiency correction factor is given by $\alpha_{m}$, which consequently resulted in the effects of multiple ion species largely not being captured in this study with $\alpha_{m}=0.9$.

$$
\begin{gathered}
\eta_{m}=\frac{\dot{m}_{i}}{\dot{m}_{p}}=\frac{I_{b}}{e} \frac{M}{\dot{m_{p}}} \\
\eta_{m}=\alpha_{m} \frac{I_{b}}{e} \frac{M}{\dot{m_{p}}} \\
\alpha_{m}=\frac{1+\frac{1}{2} \frac{I^{++}}{I^{+}}}{1+\frac{I^{++}}{I^{+}}}
\end{gathered}
$$

Applying this methodology without the correction factor to the Variable Magnet Length Cusped Field Thruster (VML-CFT) validation case of Ma et al. [9], resulted in a mass utilization efficiency of $89 \%$, which correlates well to the results of Matlock [17] in a comparable study on the DCFT, where the mass utilization efficiency was experimentally measured to be $87 \%$.

The plume divergence efficiency is not taken into account in the present model. Experimental testing would be required to accurately determine the divergence angle. A divergence angle of $\theta_{g}=60^{\circ}$ can be assumed based on Keller et al. [3], and this is supported by the measured divergence angle of $56^{\circ}$ of the DCFT by Matlock [17]. The divergence efficiency can be defined by Löb and Freisinger [18] by

$$
\eta_{\text {div }}=\frac{\left(1+\cos \theta_{g}\right)}{2}
$$

The assumptions made regarding the acceleration, divergence and utilization efficiencies will be applied in post processing of the MDO results, as these are factors which this study is unable to accurately define with the present methodology and will have a uniform effect on the objective parameters.

Kornfeld et al. [5] describe a simplified power balance description of the HEMP-T based on the plasma fluid theory. A one-dimensional set of equations (28 in total) to be solved simultaneously can be used to obtain a reasonable estimate of the thruster performance. As the only known values in the equation set are the probabilities to reach the channel wall at the cusp locations and these probabilities are based on the magnetic field strength, the thruster performance can be estimated through only a few parameters. These are anode potential, anode current and the ratio of magnetic field strength from the axially aligned region to where the fields radially cross the discharge channel walls, i.e., the magnetic mirror strength. It is also important to note that the ratios (described in Kornfeld et al. [5]) of power transferred to excitation, ionization and thermalization are only estimations. It is also stated that inclusion of angular and ionization efficiencies could allow for predictions of total thruster efficiency. 
The following description was derived from the equation system presented by Kornfeld et al. [5], where a full description of the power model can be found.

The operational principle of the CFT is based on the magnetic mirror effect due to the Permanent Periodic Magnets (PPM), which reduces losses from electrons impinging on the discharge chamber walls. This is because the particle is acted on by the Lorentz force, where the magnetic field does not have an impact on the particle, rather an axial force relative to the magnetic field acts on the particle in the opposite direction defined by the Lorentz force in a cylindrical field $[2,13,15,19]$.

$$
F_{z}=-\frac{m v_{\perp}^{2}}{2 B} \nabla_{\|} B
$$

When the particle is moving through a magnetic field of increasing strength, the magnetic moment is constant, which means that the magnetic moment at the high field and at the low field can be balanced by equating the magnetic moment of two points, where the subscript 0 indicates the low field and $m$ the high field.

$$
\frac{v_{\perp 0}^{2}}{B_{0}}=\frac{v_{\perp m}^{2}}{B_{m}}
$$

Through the conservation of the particle kinetic energy, the velocity of the particle is defined by parallel and perpendicular velocity components.

$$
v_{\|}=\left[\frac{2}{m}(K-\mu B)\right]^{1 / 2}
$$

The magnetic mirror effect is not entirely efficient, with the direction of the velocity vector needing to be within an acceptance angle in order to escape the mirror effect. This is established between the difference of the high and low magnetic field strength regions, as derived below [19]:

$$
\begin{gathered}
\frac{B_{m}}{B_{0}}=\frac{v_{\perp 0}^{2}+v_{\| 0}^{2}}{v_{\perp 0}^{2}} \\
=\frac{1}{\sin ^{2} \theta_{m}} \\
\theta_{m} \leq \sin ^{-1}\left(\sqrt{\frac{B_{0}}{B_{m}}}\right)
\end{gathered}
$$

This is then used to calculate the cusp arrival probabilities of the electrons, which is used as the basis for thruster performance using the one dimensional power equations [5]:

$$
p_{c} \equiv \frac{2 \pi\left(\int_{0}^{a_{c}} \sin (\theta) d \theta\right)}{4 \pi}
$$

The cusp arrival probabilities are directly related to the accuracy of the simulated magnetic topologies of the thruster, which is calculated by means of two-dimensional electromagnetic field analysis.

Shown in Figure 1 is the process chain followed to produce decision variables and delivery of the objective functions. The decision variables are assessed to assure the geometry is physically possible before they are passed onto the software ANSYS Maxwell [20] for model construction and the computation of the magnetic field. Magnetostatic analysis is then performed to calculate the magnetic field strength topologies (Figure 2), and then an electrostatic analysis is performed to determine the potential at the thruster exit and in the plume. 


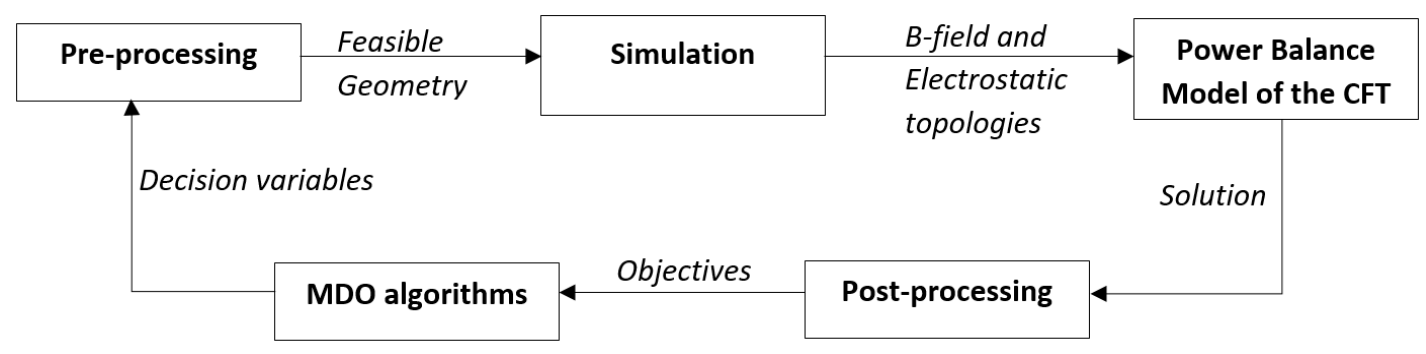

Figure 1. Multi-objective design optimization (MDO) process chain.

\begin{tabular}{|c|c|}
\hline B [tes 1a] & \\
\hline & 0.6810 \\
& 0.6130 \\
& 0.5450 \\
& 0.4769 \\
& 0.4089 \\
& 0.3409 \\
0.2729 \\
0.1368 \\
0.0688 \\
\hline
\end{tabular}

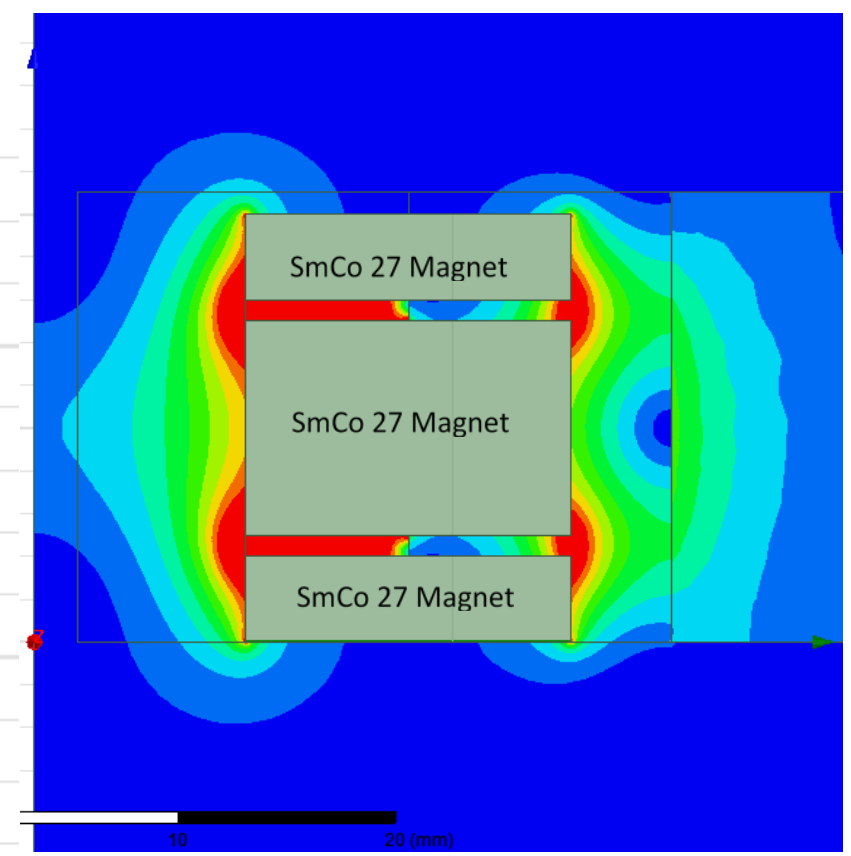

Figure 2. Magnetic field strength topology of non-dominated solution, $\mathrm{S}_{1}$, from the results of the simulation.

The data is extracted from the magnetic topology to compute the cusp arrival probability for each location throughout the thruster. These conditions are subsequently passed to be used in the power balance calculation. The resultant solutions from this are post-processed to deliver the objectives and assess if they lie within the set of physical constraints. They are then submitted to the MDO algorithms for evaluation. This cycle repeats as more designs are produced and evaluated as per the set criteria.

Design optimization is performed by employing evolutionary algorithms [21,22]. In particular, use is made of the elitist non-dominated sorting genetic algorithm [23] assisted by surrogate modelling. It is a population-based approach where the candidate solutions in the population pool evolve over generations. A population size of $N=64$ is used in this study to be evolved over 40 generations for the initial MDO study employing simulation, and that of $N=100$ over 100 generations in the MDO run performed solely based on prediction from surrogate models. These values have been chosen to sufficiently explore the design space for a three-objective design problem with five decision variables within reasonable computational effort. Recombination operators are applied to the previous generation's decision variable values to create offspring. A simulated binary crossover and polynomial mutation are used as recombination operators at a given probability (1.0 and 0.1 , respectively, in this study) with a specified distribution index (10 and 20, respectively) [22]. The use of a strongly elitist non-dominated sorting genetic algorithm always retains the best solutions across generations. This means that there is no incentive for parents to survive but to use the crossover mutation to broaden the search for better solutions; hence the crossover probability is given by 1.0. Surrogate 
modelling [24] is commissioned to estimate the possible values of the objectives and constraints in an inexpensive manner, imitating the behaviour of the solutions from the magnetic and power analyses with meta-models characterized by appropriate mathematical functions based on the magnetostatic, electrostatic and power balance simulations. Different surrogate models are employed to best predict the thruster performance with a minimum error threshold satisfied, as discussed below. The surrogates are constructed by using a fraction ( $90 \%$ in this study) of the actual solutions calculated from the magnetic and power distribution models to prevent over fitting, whereas the remainder $(10 \%)$ of the evaluated solutions are used to assess the performance of the surrogate models [22]. Surrogates for each of the objective and constraint functions are trained by using a subset of the archive, which is created by selecting the solutions closest to the centroids of the $k$ clusters obtained through $k$-means clustering [25]. Multiple surrogate models are considered and evaluated: quadratic response surface model, [26] artificial neural network (ANN) models including the radial basis function network [27] and multilayer perceptron model, [28] which are single-layer and feed forward types of ANN models, respectively, and Kriging model based on Gaussian process regression [29]. The mean squared error (MSE) in the actual and predicted values of the objectives and constraints is calculated for the remaining $(10 \%)$ solutions and used as the measure to validate the surrogate models. Prediction from the best surrogate model with a minimum error is adopted to replace simulation analysis, only if the MSE is within a threshold value of $5 \%$ for all objective and constraint functions, which has been set to effectively allow reasonable investigation by striking a balance between prediction accuracy and computational cost, and the distance to the closest point in the archive is smaller than 5\% [22]. The advantage of the MDO approach undertaken in the present study is the ability to maintain multiple objectives and perform optimization to aim at all the objectives simultaneously, which is the primary virtue of MDO. This removes the no need for weighting on the objectives to convert a multiple-objective optimization problem to a single objective one, as required for a single-objective optimization framework.

The three objective functions used to evaluate design performance are thrust $T$, efficiency $\eta_{t}$ and specific impulse $I_{s p}$ (the efficiency term with the subscript $t$ is somewhat of a misnomer here, usually descriptive of total efficiency, and it must be stated that the model presented in this paper does not take into consideration plume divergence losses or an accurate representation of acceleration efficiencies). The $\eta_{t}$ term is comprised of the measures of efficiencies within the thruster model, that is, the beam efficiency $\eta_{b}$, the mass utilization efficiency $\eta_{u}$ and the voltage efficiency $\eta_{v}$. The decision variables and their input ranges are chosen to represent the main design factors investigated in this study (shown in Algorithm 1) are $\Phi_{a}(\mathrm{~V}), I_{a}(\mathrm{~A}), m_{f}(\mathrm{sccm})$, Inner Magnetic Radius (IMR) (mm), and Outer Magnetic Radius (OMR) (mm). The optimization statement can be expressed as follows (the negative sign for the objective functions denotes a maximization problem). The decision parameters $\Phi_{a}(\mathrm{~V}), I_{a}(\mathrm{~A})$, and $m_{f}(\mathrm{sccm})$ relate to the objective functions through Equations (1)-(5) and the initial conditions of the CFT at the anode. The magnetic radii, IMR and OMR, are related to the objective functions through the one-dimensional simplified power balance model. The radii effect the strength of the magnetic field in the high and low regions which is used to solve the power balance model, describing the plasma potentials, cusp potentials, ionization source currents, and electron and ion currents across plasma cells that divide the discharge chamber length in the simplified model. From this determination, the beam and grid efficiencies is made, calculating power losses at the anode, cusps and due to ionization and excitations, which impacts on each separate objective function.

\begin{tabular}{ll}
\hline Algorithm 1 Optimization parameters \\
\hline Maximize: & $T, \eta_{t}, I_{s p}$ \\
Subject to: & $0 \leq \Phi_{\mathrm{a}}(\mathrm{V}) \leq 1000$ \\
& $0 \leq \mathrm{I}_{\mathrm{a}}(\mathrm{A}) \leq 10$ \\
& $0.2 \leq \mathrm{m}_{\mathrm{a}}(\mathrm{sccm}) \leq 50$ \\
& $2 \leq \mathrm{IMR}(\mathrm{mm}) \leq 50$ \\
& $2 \leq \mathrm{OMR}(\mathrm{mm}) \leq 50$ \\
\hline
\end{tabular}


To validate this approach, a model of the experimental setup of the CFT thrusters from the available literature are created using ANSYS Maxwell. The selected experimental model to validate the procedure is the VML-CFT from Ma et al. [9]. This model is considered because of its detailed account of the physical dimensions and material properties of the thruster and its subsequent analysis of the results gives a robust range of observation points that can be modeled and compared.

From the simulated model, two analyses are performed; firstly, a magnetostatic solver is used to determine the magnetic field strength at the cusps along the inner engine radius/wall. This magnetic strength is compared to the magnetic strength at the centerline of the engine, where the particles are in the observed loss cone $[17,19]$. The difference between the magnetic field strengths is used to determine the Magnetic Mirror Ratio at each cusp. This is then used to calculate the cusp arrival probabilities at each magnetic cusp. The cusps arrival probabilities for the validation model at the four magnetic cusps are shown in Table 1.

Table 1. Magnetic cusp arrival probabilities.

\begin{tabular}{cc}
\hline Cusp Probability & \\
\hline $\mathrm{C}_{1}$ & 0.0167 \\
$\mathrm{C}_{2}$ & 0.0030 \\
$\mathrm{C}_{3}$ & 0.0259 \\
$\mathrm{C}_{4}$ & 0.0037 \\
Beam Efficiency & 0.8001 \\
\hline
\end{tabular}

The calculated cusp probabilities accuracy is difficult to determine through a lack of available reference data. From the literature, it is expected that the cusp probabilities will differ significantly from the experimental probing of the thrusters. From the data of Kornfeld, et al. [5], from which the simplified HEMP-T model originates, the cusp probabilities observed in thruster Demonstration Model (DM) 9.2 and DM 10 appear to correlate well with the exception $C_{2}$ and $C_{4}$ which is expected to be significantly larger then measured. The cusp arrival probabilities are expected to be below $5 \%$ of electrons with a noted maximum at the third cusp. While this is reflected in the simulations, the validation of the cusp arrival probabilities is significantly underpinned by the overall accuracy of the simplified HEMP-T model by Kornfeld, et al. [5], and in comparison to experimental research (as documented in Table 2) as well as in comparison to the preceding results by Muffatti and Ogawa [15], especially in relation to efficiency.

Table 2. Comparison of experimental and simulated results.

\begin{tabular}{|c|c|c|c|c|c|c|c|}
\hline \multirow{2}{*}{$\begin{array}{l}\text { Propellant } \\
\text { Mass Flow, } \\
m_{a} \text { (sccm) }\end{array}$} & \multicolumn{3}{|c|}{ Ma, et al. [9] } & \multicolumn{4}{|c|}{ Present } \\
\hline & $\begin{array}{c}\text { Total } \\
\text { Efficiency }\end{array}$ & $\begin{array}{c}\text { Specific } \\
\text { Impulse, } I_{s p}(\mathbf{s})\end{array}$ & $\begin{array}{l}\text { Thrust } \\
(\mathrm{mN})\end{array}$ & $\begin{array}{c}\text { Total } \\
\text { Efficiency }\end{array}$ & $\begin{array}{c}\text { Specific } \\
\text { Impulse, } I_{s p}(\mathbf{s})\end{array}$ & $\begin{array}{l}\text { Thrust } \\
(\mathrm{mN})\end{array}$ & $\begin{array}{l}\text { Thrust } \\
\text { Error \% }\end{array}$ \\
\hline 10 & 0.200 & 1971 & 19 & 0.2944 & 2368 & 22.83 & 16.78 \\
\hline 20 & 0.330 & 2178 & 42 & 0.3913 & 2364 & 45.58 & 7.85 \\
\hline 30 & 0.345 & 2282 & 66 & 0.3839 & 2356 & 68.12 & 3.11 \\
\hline 40 & 0.350 & 2256 & 87 & 0.3850 & 2345 & 90.41 & 3.77 \\
\hline 50 & 0.348 & 2324 & 112 & 0.3525 & 2317 & 111.69 & 0.28 \\
\hline
\end{tabular}

The second analysis performed is an electrostatic simulation, which is used to calculate the potential drop in the plume. Using the electrostatic analysis results, the difference between the cusp potentials at the plume cell and the thruster exit can be more accurately predicted in line with observations made in the literature. The simplified HEMP-T model Kornfeld et al. [5] tended to over predict the potential in the plume region, which gave rise to efficiency inaccuracies when calculating the grid efficiency and acceleration efficiency as well as over predicting the beam current, $I_{b}$. The results of the electrostatic thruster solution are shown in Table 3. It shows the discrepancies between the HEMP-T simplified model assumptions and the observed potential behavior demonstrated in the simulation. 
Table 3. Electrostatic plasma cell solution.

\begin{tabular}{cc}
\hline Magnetic Cusp Cell Potential & Model Potential \\
\hline$\Phi_{1}($ Plume Potential) & $36.54 \mathrm{~V}$ \\
$\Phi_{2}$ (Exit Cell) & $373 \mathrm{~V}$ \\
$\Phi_{3}$ (Middle Cell) & $584 \mathrm{~V}$ \\
$\Phi_{4}$ (Anode Cell) & $387 \mathrm{~V}$ \\
Grid Efficiency & 0.9079 \\
\hline
\end{tabular}

The expected outcomes of the internal magnetic cell potentials should be in line with the voltage applied to the anode, $500 \mathrm{~V}$ for the VML-CFT, in accordance with Kornfeld et al. [5]. The variation is likely due to electrostatic model setup and the over prediction of the initial conditions using the nonlinear least squares method.

The comparison between the results of Ma, et al. [9] for a VML thruster with magnetic cell length ratios of 2:9:0.5 is shown in Table 2. This specific experimental model represented the peak performance characteristics of the thruster. The comparison of the results is very close to the experimental result. Except for the lowest mass flow rate where the total efficiency has an approximate $10 \%$ error, the efficiencies are only slightly higher than the measured result with a trend toward convergence with higher propellant mass flow rates. The thrust is measured from two different methods, from Kornfeld et al. [5] and then from the method described by Keller et al. [3]. These calculations are both good predictions of the thrust and appear to validate the approach undertaken. With very close predictions for propellant mass flow rates between 20 and $40 \mathrm{sccm}$, it would be appropriate to restrict the considered design MDO process between these limits. The model was a poor predictor of specific impulse. This appears to be owing to the sensitivity of the $I_{s p}$ calculation to the small thrust inaccuracy and as a result the $I_{s p}$ does not see the desired variation with mass flow rate.

Variance-based global sensitivity analysis is performed to investigate the influence of each decision variable $x_{i}$ as input (i.e., design parameter) on the objective function $y$ as output (i.e., thruster performance parameter). A numerical procedure (Sobol's variance decomposition [30]) is employed to derive the sensitivity indices, facilitated by surrogate modelling [24]. Input matrices $X$ of a base sample quantity of 10,000 and multiple columns for the decision variables are built by using quasi-random numbers [30] within the range for each variable. Output vectors $Y$ are obtained by forwarding the input matrices to the surrogate model that is of the greatest prediction accuracy. The first-order indices $S_{i}$ and total-effect indices $S_{T i}$ are calculated by the method outlined in Reference [31], defined as:

$$
\begin{gathered}
S_{i}=\left[E\left(Y \mid X_{i}\right)\right] / V(Y) \\
S_{T_{i}}=1-V\left[E\left(Y \mid X_{-i}\right)\right] / V(Y)
\end{gathered}
$$

The CFT model developed in ANSYS Maxwell is designed to be as simple as possible for use in the MDO calculations. This is due to the computationally expensive nature of this process and to facilitate the identification of the relationships between the output objectives and the decision variables. The model is set up for two-dimensional Maxwell simulation, and the CFT is modeled by assuming axis symmetry at the engine axis. The mesh is created with 9000 elements. The thruster itself features a consistently straight chamber, three magnet stages with spacers (doubling as field guides), and the thruster housing. Used in the simulation are Samarium-Cobalt (SmCo) 27 megaguass-oersteds (MGOe) magnets stacked with reversing polarity and the thruster housing material used is Al 6061-T6. The spacers are made from pure iron due to the high conductivity of the material as well as to improve commonality with other HEMP-T/CFT designs $[3,8,11,16]$. Several geometric constraints were implemented to restrict the scope and output of the design space, including that imposed on the inner and outer radii, i.e., IMR and OMR. The basic physical constraints implemented into the model are simple in nature, that is, rejection of geometries that overlap in regions as infeasible solutions in MDO. The somewhat arbitrary limitations selected are those for the inner thruster channel geometry 
( $2 \mathrm{~mm}$ radius) as a lower limit and the upper limit (50 mm radius) to remain approximately within the limits of the standard CubeSat form. Further restrictions are placed on the utilization of propellant regarding anode current. The upper limit is set in the pre-processing stage where $I_{b}$ is replaced with $I_{a}$ and cannot produce a value exceeding 1 , because the only ion species under consideration are the singly charged variety [2].

\section{Results}

Multi-objective optimization based on evolutionary algorithms is performed for up to 40 generations with a population size $N$ of 64 . Surrogate models were constructed after 40 generations to be employed in a surrogate-only optimization with a population size of $N=100$ evolved over 100 generations. It has been performed to fully explore the design space at reduced computational cost by substituting simulations with surrogate prediction entirely. The surrogate prediction errors are $0.8 \%$ for $T, 1.6 \%$ for $\eta_{t}$, and $1.2 \%$ for $I_{s p}$. Figure 3 displays the non-dominated individuals obtained thus, where a vector of the objective functions $f\left(x^{\prime}\right)$ is said to be non-dominated if there exists no other vector $f(x)$ that satisfies $f(x) \leq f\left(x^{\prime}\right)$ with $f_{i}(x)<f_{i}\left(x^{\prime}\right)$ for at least one index $i \in(1,2,3)$ [32]. Also, included in these plots are 4 individuals $\left(\mathrm{S}_{1-4}\right)$ that have been selected to investigate the key design factors and associated context of their creation. $S_{1}, S_{2}$, and $S_{3}$ are non-dominated solutions that perform the best with respect to each design criterion, that is, $S_{1}$ in $T, S_{2}$ in $\eta_{t}$, and $S_{3}$ in $I_{s p} . S_{4}$ is a suboptimal solution, characterized by low performance in all 3 objective categories.

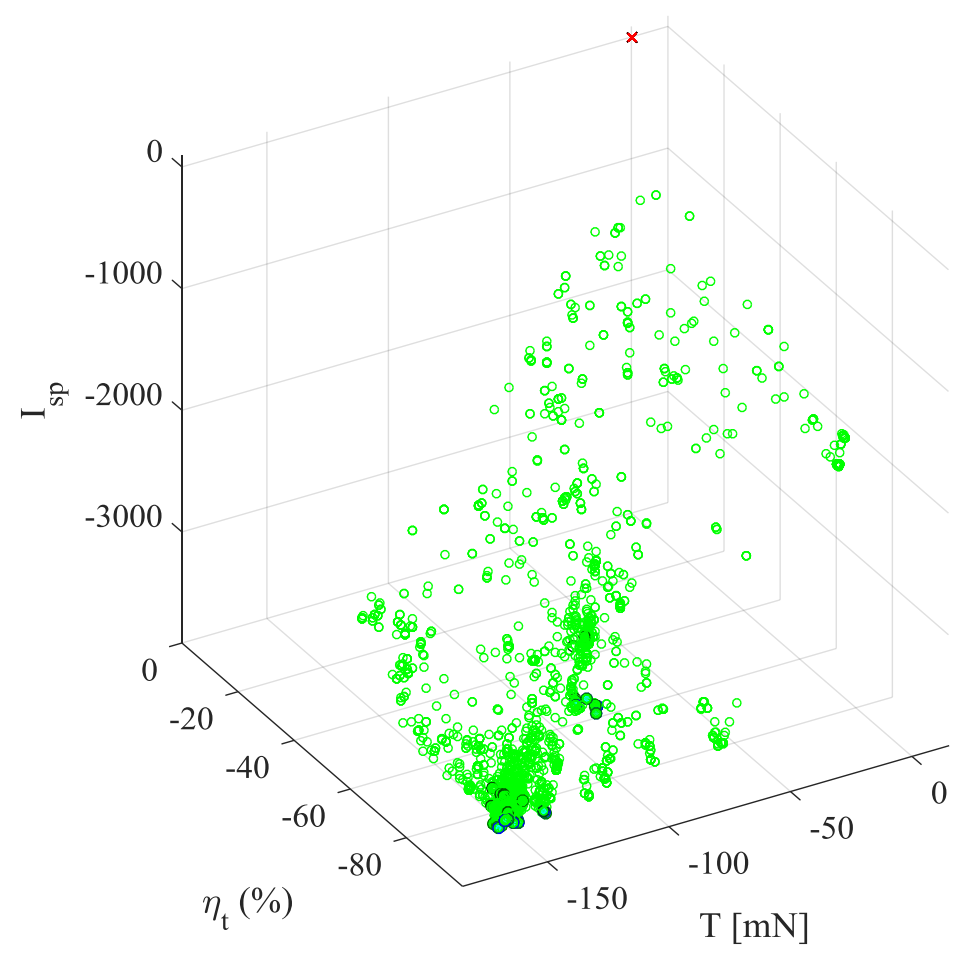

Figure 3. Optimization results from MDO using analysis (at 40th generation with $N=64$ ). The green points indicate the feasible geometries, the blue points are non-dominated solutions and the red crosses represent unfeasible solutions.

The feasible solutions from the MDO coupling ANSYS Maxwell analysis are presented in Figure 3. These results are used to build surrogate models. Figure 4 displays the solutions from an MDO study performed by solely relying on surrogate prediction. It shows a uniform distribution of solutions along the Pareto optimal front. The smoothness of the Pareto optimal front indicates the robust nature of the surrogate modeling optimization using a population size of 100 evolved over 100 generations. It means that the solution converges to a globally optimum point and the process is unlikely to have 
excluded feasible non-dominated results. The quality of the optimization is a complex concept but can be assessed by the metrics of performance defined by Zitzler et al. [33]. Figure 4 demonstrates reasonable performance of the MDO based on the distance between the non-dominated solutions and the Pareto optimal front, a uniform distribution of feasible solutions, and a wide range of solutions are explored for each objective [33].

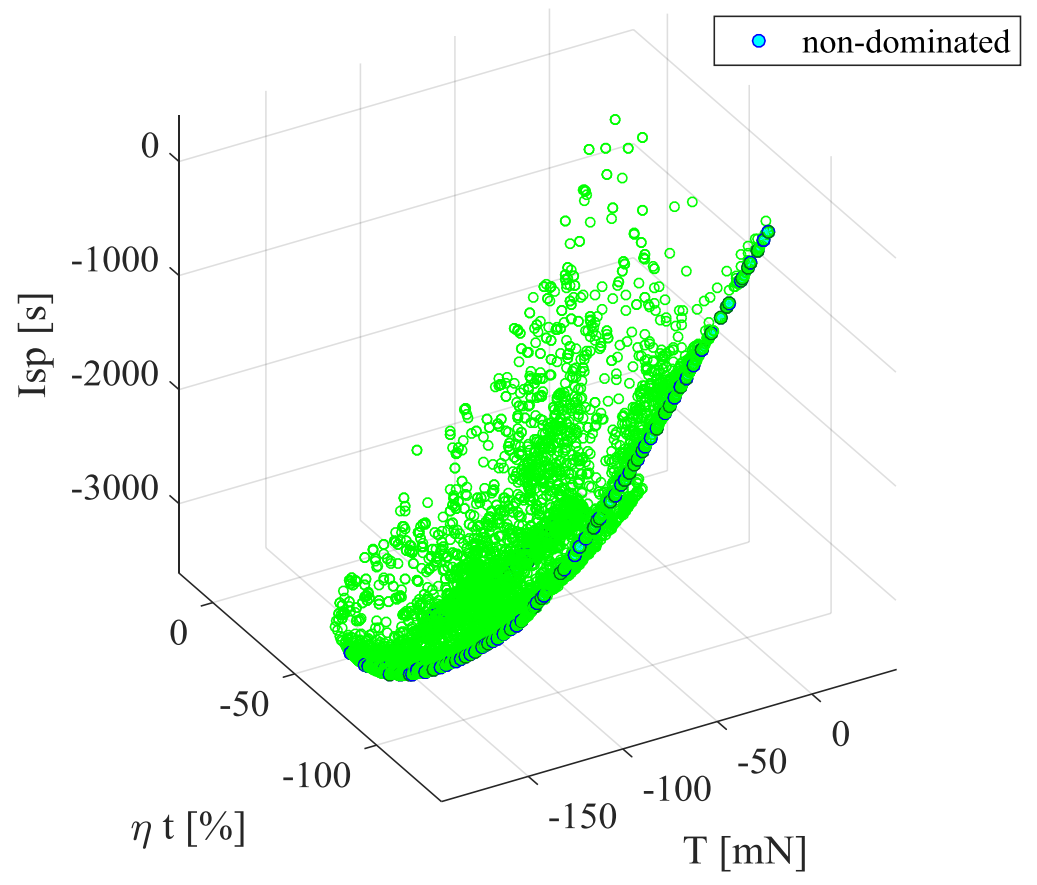

Figure 4. Optimization results from surrogate-only MDO evaluations (at 100th generation with $N=100$ ). The green points indicate the feasible geometries, and the blue points are non-dominated solutions.

\section{Sensitivity Analysis}

Figure 5 displays the first-order sensitivity indices $S_{i}$ and the total-effect indices $S_{T i}$. They indicate the main and overall effects of the input parameters (decision variables) respectively, i.e., $\Phi_{a}, I_{a}, m_{a}$, IMR, and OMR on the output parameters (i.e., objective functions), namely thrust $T$, efficiency $\eta_{t}$, and specific impulse $I_{s p}$. The difference between the total-effect index $S_{T i}$ and the first-order index $\mathrm{Si}$ is indicative of the degree of the influence of the decision variable in combination with other decision variables (i.e., interactions) on the objective functions [30]. If the decision variables are characterized by the sum of both first-order and total-effect indices being near unity (i.e., $\Sigma S_{i} \approx 1$ and $\Sigma S_{T i} \approx 1$ ), this indicates that the effects of individual decision variables are linearly additive. From Figure 5 it is observed that most of the influence on the design is based on 4 critical design parameters; OMR, IMR, $I_{a}$, and $m_{a} . I_{a}$ has the most influence on thrust $T$ and specific impulse $I_{s p}$, while OMR is dominant in total efficiency. From Figure 5, it is evident that the decision variables exhibit similar influence across the three objectives as to be expected from Equation (4).

The first-order indices are shown in Table 4, demonstrating the large influence of IMR, OMR, $I_{a}$, and $m_{a}$ across the objective functions with the anode potential exerting significantly lower influence over the output parameters. The summation of the first-order effects on thrust is 0.2386 while that of the total-effect indices on thrust (Table 5) is 2.137, indicative of the highly nonlinear relationship between the input and output parameters. The total-effect indices tabulated in Table 5 indicate that the indices follow the same order of influence but with all variables increasing significantly in magnitude due to the involvement of other design parameters. 


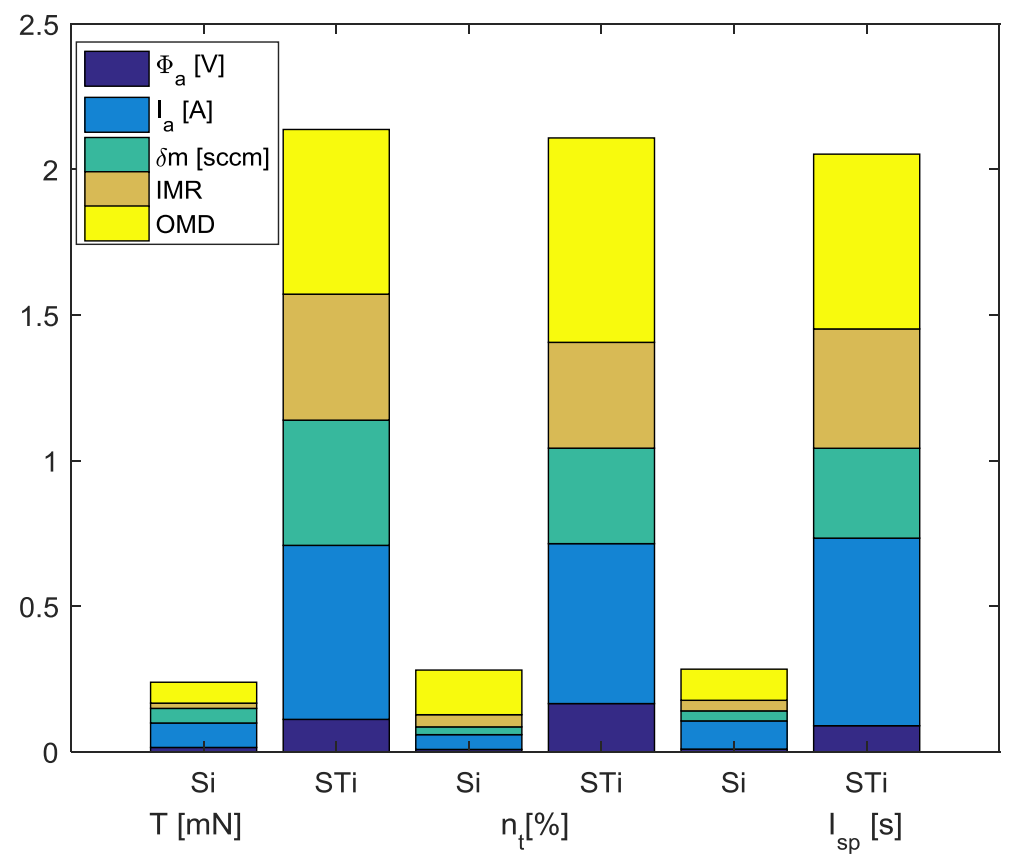

Figure 5. Sensitivity of performance parameters to design parameters.

Table 4. First-order indices for influences of design parameters on performance parameters.

\begin{tabular}{cccccc}
\hline Parameter & $\boldsymbol{\Phi}_{\boldsymbol{a}}$ & $\boldsymbol{I}_{\boldsymbol{a}}$ & $\boldsymbol{m}_{\boldsymbol{a}}$ & IMR & OMR \\
\hline$T$ & 0.015 & 0.084 & 0.050 & 0.018 & 0.072 \\
$\eta_{t}$ & 0.007 & 0.051 & 0.026 & 0.042 & 0.153 \\
$I_{s p}$ & 0.009 & 0.097 & 0.034 & 0.036 & 0.167 \\
\hline
\end{tabular}

Table 5. Total-effect indices for influences of design parameters on performance parameters.

\begin{tabular}{cccccc}
\hline Parameter & $\boldsymbol{\Phi}_{\boldsymbol{a}}$ & $\boldsymbol{I}_{\boldsymbol{a}}$ & $\boldsymbol{m}_{\boldsymbol{a}}$ & IMR & OMR \\
\hline$T$ & 0.111 & 0.597 & 0.430 & 0.433 & 0.565 \\
$\eta_{t}$ & 0.165 & 0.549 & 0.328 & 0.363 & 0.702 \\
$I_{s p}$ & 0.089 & 0.644 & 0.308 & 0.409 & 0.600 \\
\hline
\end{tabular}

The large influence that $m_{a}$ and $I_{a}$ have on $\eta_{t}$ is not entirely unexpected due to the highly interrelated nature of total efficiency calculation. The anode current is strongly dependent on the mass flow rate, i.e., higher mass flow results in higher thrust as per Equation (1), but considering these decision variables independently would allow to assess the degree of their influence on the objectives and offer flexibility in identifying the optimal design. These results indicate the importance of matching these two parameters to achieve nominal conditions. However, it is important to note that other measures for the efficiency such as the acceleration efficiency and beam divergence losses are not taken into consideration in this model and would be required to be estimated from existing experimental research. The parameter influences on $I_{s p}$ continue this trend, subject to the considerable influence of the anode current, mass flow rate and OMR, which correlates to the results of Muffatti and Ogawa [15]. This is reinforced by the observations regarding thrust, where anode potential has little influence compared to the other decision variables. Given the interrelated nonlinear nature of the problem, the sensitivity analysis supports the use of the optimization process on the parameters to better understand the concept and underlying mechanism, and improve the thruster performance.

Figures 6 and 7 are parallel coordinate plots drawn for the non-dominated solutions identified in the MDO study using analysis at the 40th generation and that with surrogate prediction at the 100th 
generation, respectively. They are utilized to identify the trends between the parameters. The plots are dependent on the order and scale of the variables where the pattern of the lines indicate a cooperating (parallel) or counteracting (crossing) trend between the parameters [34]. The parameters are arranged with the performance parameters first followed by the anode parameters because of the trends found in literature [3], which suggested the anode conditions have greater influence on the performance of the thruster. Further analysis is required to compare the decision parameters effect on each other. Figure 6 shows a positive, cooperating relationship between the thrust $T$, and efficiency $\eta_{t}$. However, there is a counteracting trend between efficiency $\eta_{t}$, and specific impulse $I_{s p}$. In Figure 7 , the relationship between specific impulse $I_{s p}$, and anode potential $\Phi_{a}$, is considered counteracting and negative. The trend of the subsequent design parameters, from anode potential $\Phi_{a}$, to anode current $I_{a}$, is shown as cooperating and positive. A specific trend between anode current $I_{a}$, and mass flow $m_{a}$, is not discernible. Counteracting negative trends are also observed between mass flow $m_{a}$, and IMR as well as between IMR and OMR in Figures 6 and 7.

The parallel coordinate plots also show that the variables are decisive, in that the variables must be more decisive if the lines gather, as occurs for IMR and OMR. For anode potential $\Phi_{a}$, and mass flow $m_{a}$, the performance could be further enhanced by expanding (loosening) the limits, as the upper limits have been reached for non-dominated solutions for these parameters.

Figures 3, 5 and 6 have been produced after the evolutionary process of the population consisting of 64 individuals over 40 generations. Displayed in Figure 3 are the non-dominated solutions from the MDO process, along with evaluated feasible design points. Of the non-dominated solutions, 3 solutions with the highest values for the objective parameters, thrust $T$, efficiency $\eta_{t}$, and specific impulse $I_{s p}$, are selected from the 39 generated non-dominated solutions, along with single suboptimal design for comparison as shown in Table 6.

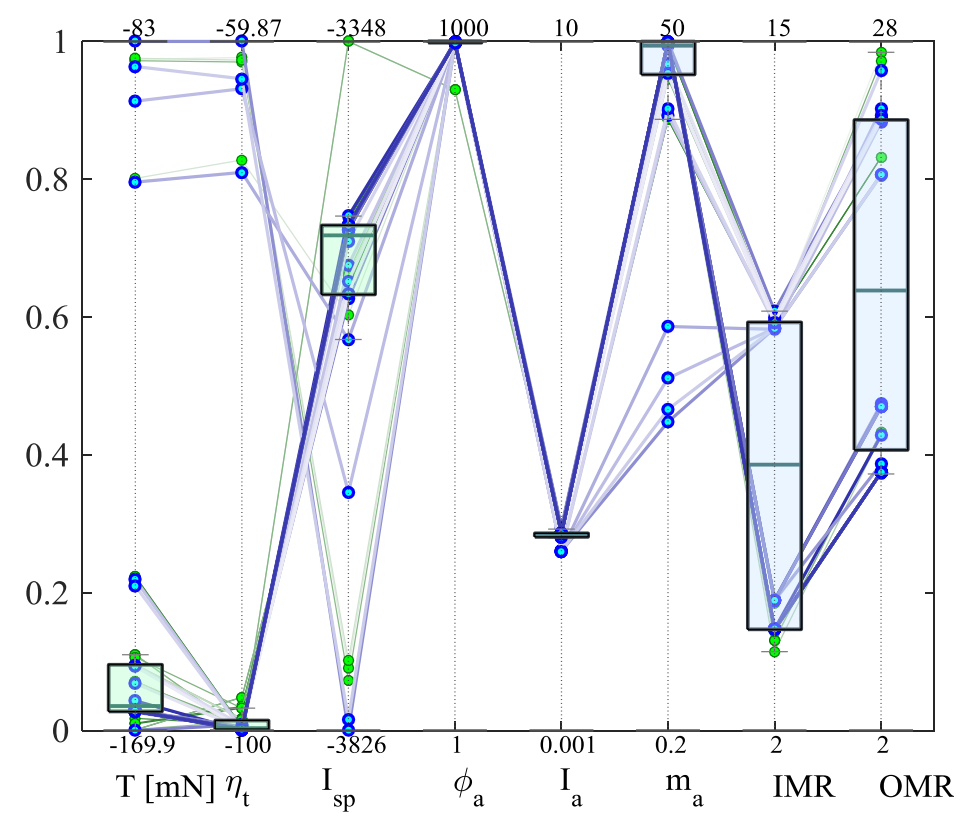

Figure 6. Parallel coordinate plot for MDO using analysis (at 40th generation with $N=64$ ). The green points and lines indicate feasible solutions, the blue point and lines indicate non-dominated solutions and frames indicate the range of solutions that have the same relationship with the corresponding objective and decision variables. 


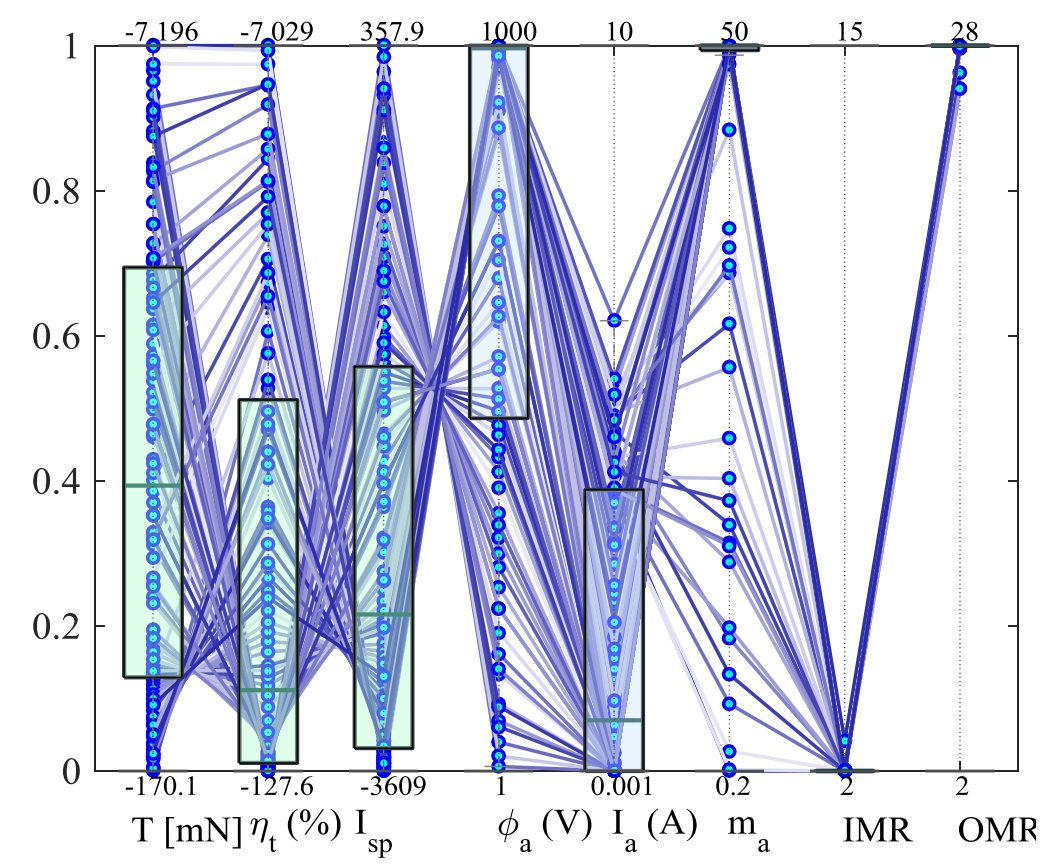

Figure 7. Parallel coordinate plot for surrogate-only MDO (at 100th generation with $N=100$ ). The frames indicate the range of solutions that have the same relationship with the corresponding objective and decision variables.

Table 6. Selected design configurations.

\begin{tabular}{ccccccccc}
\hline Solution & $\boldsymbol{T} \mathbf{( M n})$ & $\boldsymbol{\eta}_{\boldsymbol{t}}(\mathbf{\%})$ & $\boldsymbol{I}_{\boldsymbol{s} \boldsymbol{p}}(\mathbf{s})$ & $\boldsymbol{\Phi}_{\boldsymbol{a}}(\mathbf{V})$ & $\boldsymbol{I}_{\boldsymbol{a}}(\mathbf{A})$ & $\boldsymbol{m}_{\boldsymbol{a}}(\mathbf{s c c m})$ & $\mathbf{I M R}(\mathbf{m m})$ & OMR $(\mathbf{m m})$ \\
\hline $\mathrm{S}_{1}$ & 169.9 & 99.6 & 3526 & 999.9 & 2.94 & 49.98 & 9.91 & 25.10 \\
$\mathrm{~S}_{2}$ & 166.2 & 99.9 & 3469 & 998.4 & 2.83 & 49.69 & 3.91 & 13.13 \\
$\mathrm{~S}_{3}$ & 82.9 & 59.8 & 3825 & 997.8 & 2.61 & 22.51 & 9.59 & 22.97 \\
$\mathrm{~S}_{4}$ & 27.4 & 45.2 & 1291 & 153.3 & 2.49 & 21.98 & 3.83 & 25.45 \\
\hline
\end{tabular}

The first selected configuration $S_{1}$ exhibits the largest thrust of all the designs. This design falls on the upper limit of both mass flow and anode potential decision variables. It also has the largest anode current of the non-dominated designs. The second selected point $S_{2}$ has the highest measured efficiency of the evaluated feasible designs. It has the lowest specific impulse of its non-dominated peers, which while being similar in value to its peers, is quite significant due to the method of calculation, as observed during the validation process of this methodology. $\mathrm{S}_{2}$ has the most dissimilar physical configuration of the designs, with the IMR much closer to the engine axis and has the smallest thickness of the permanent periodic magnets. This indicates the role of IMR in achieving high thrust densities. In comparison to $\mathrm{S}_{1}, \mathrm{~S}_{2}$ has comparable thrust, efficiency, anode potential, current and mass flow. The third selected point $S_{3}$ has the highest specific impulse of all the evaluated designs. This is at the expense of thrust and efficiency. A reduction of about $90 \mathrm{mN}$ of thrust is recorded and a reduction of about $40 \%$ efficiency is observed in comparison to its non-dominated peers. $S_{3}$ has the lowest mass flow (over 50\% reduction) and the lowest anode current and lower but comparable anode potential. The suboptimal design $S_{4}$ operates at very similar conditions to $S_{3}$. This is indicated in the results from the sensitivity analysis, where the impact of a reduced mass flow $m_{a}$ on the performance parameters is shown in Figure 5. It is also correlated by the spacecraft propulsion thrust equating to the force on a charged particle in an electric field, as per the conservation of energy.

The correction factors for mass utilization, acceleration and plume divergence efficiencies based on the experimental research of Keller et al. [3], Ma et al. [9] and Matlock [17] are shown in Table 7, based on the selected points in Table 6. For mass utilization efficiency, the study allows for the presence 
of $20 \%$ doubly charged ions, resulting in a correction factor of $\alpha_{m}=0.9$ from Equation (9). The plume divergence efficiency is based on an assumed divergence angle of $60^{\circ}[3,18]$. The acceleration efficiency requires significant measurement of the CFT, which is beyond the scope of this study. Keller et al. [3] gives a combined acceleration and divergence efficiency of $40.7 \%$, (i.e., $\eta=\eta_{\text {acc }} \cdot \eta_{\text {div }}=0.407$ ) by extrapolating the acceleration efficiency from the Faraday measurement values. This produces a more conservative estimation of the objective parameters that are comparable to previous studies.

Table 7. Adjusted selected design configurations based on acceleration, divergence efficiencies and multiply charged ions.

\begin{tabular}{cccc}
\hline Solution & $\boldsymbol{T}(\mathbf{m N})$ & $\boldsymbol{\eta}_{\boldsymbol{t}}(\mathbf{\%})$ & $\boldsymbol{I}_{\boldsymbol{s} \boldsymbol{p}}(\mathbf{s})$ \\
\hline $\mathrm{S}_{1}$ & 102.7 & 36.5 & 2131 \\
$\mathrm{~S}_{2}$ & 100.5 & 36.6 & 2098 \\
$\mathrm{~S}_{3}$ & 50.2 & 21.9 & 2313 \\
$\mathrm{~S}_{4}$ & 16.5 & 16.5 & 778 \\
\hline
\end{tabular}

The results from the MDO studies indicate a trend toward maximizing the anode potential, and mass flow. This is in agreement with some of the trends observed in the available literature [15]. The geometry for high thrust and efficiency performance appears to converge and the anode current also converges towards 2.5-3.0 A.

Examination of the decision variables in relation to the output parameters in Figures 8-11 reveals several interesting relationships. In Figure 7, the anode potential tends towards the upper bound of the design range for the best results in $I_{s p}$. However, high $\Phi_{a}$ is not a good indicator of high thrust or efficiency for the thruster, although these maximums do occur for increased values of $\Phi_{a}$. Expansion of the upper limit of the anode potential is an area that should be further explored in order to determine an upper, optimal value of anode potential, and identify the point at which it starts to exert adverse effects on the objective functions.

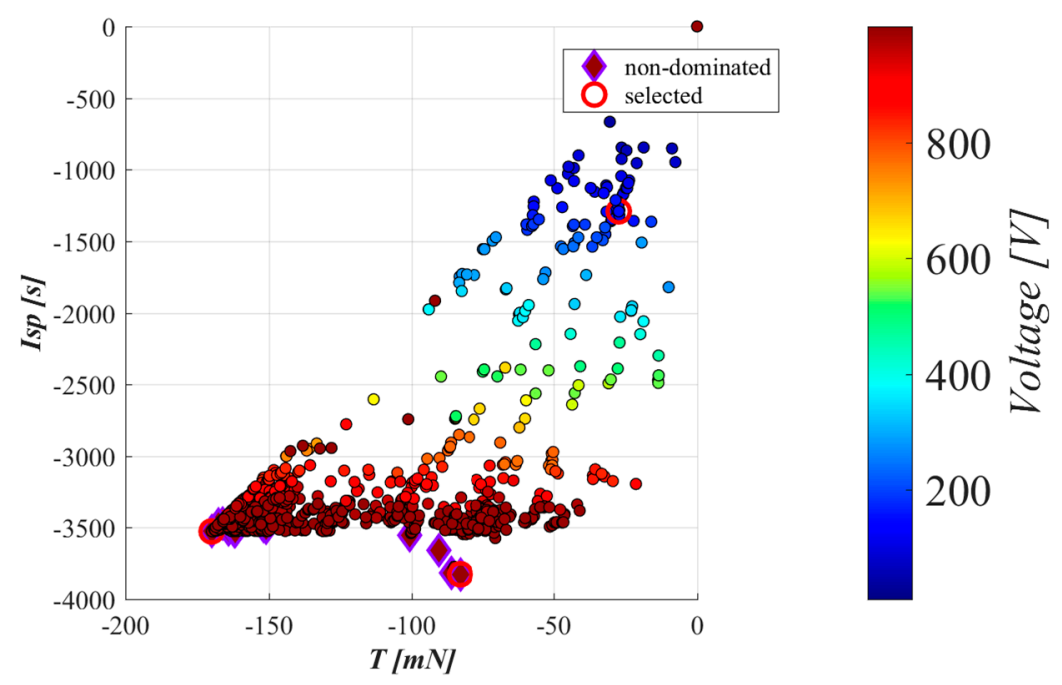

Figure 8. Anode potential color map of the feasible solutions with respect to thrust $T$ and specific impulse $I_{s p}$ (the selected and non-dominated solutions are highlighted). 


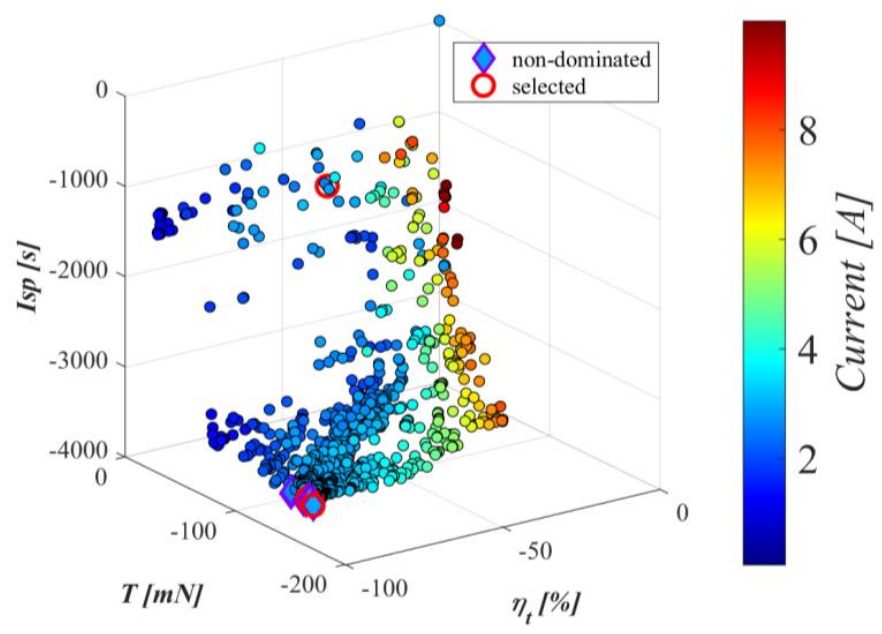

Figure 9. Anode current color map of the feasible solutions with respect to efficiency $\eta_{t}$, thrust $T$ and specific impulse $I_{s p}$.

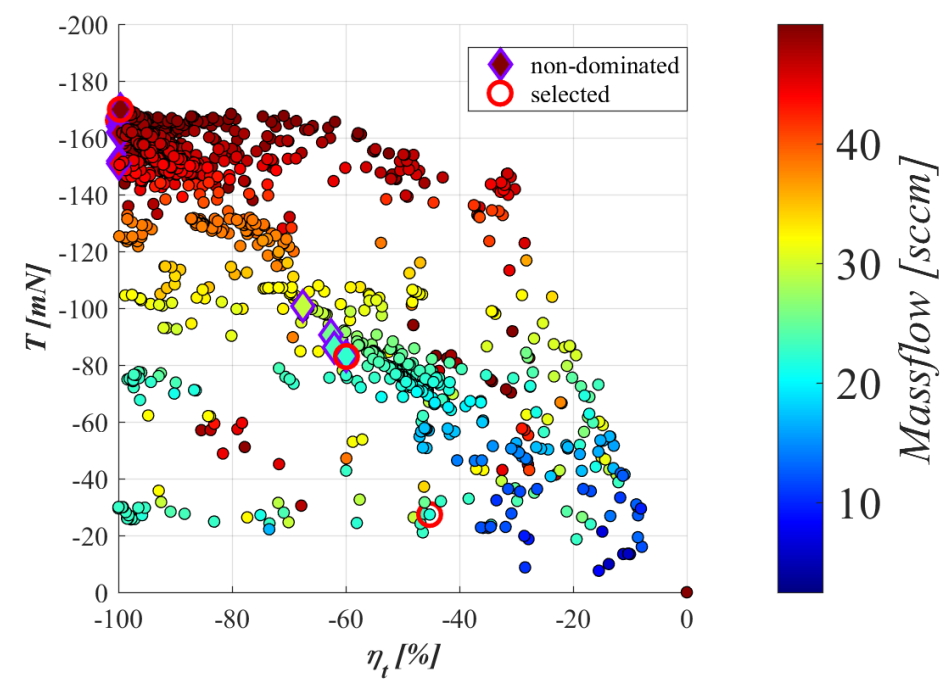

Figure 10. Mass flow color map of the feasible solutions with respect to efficiency $\eta_{t}$ and thrust $T$.

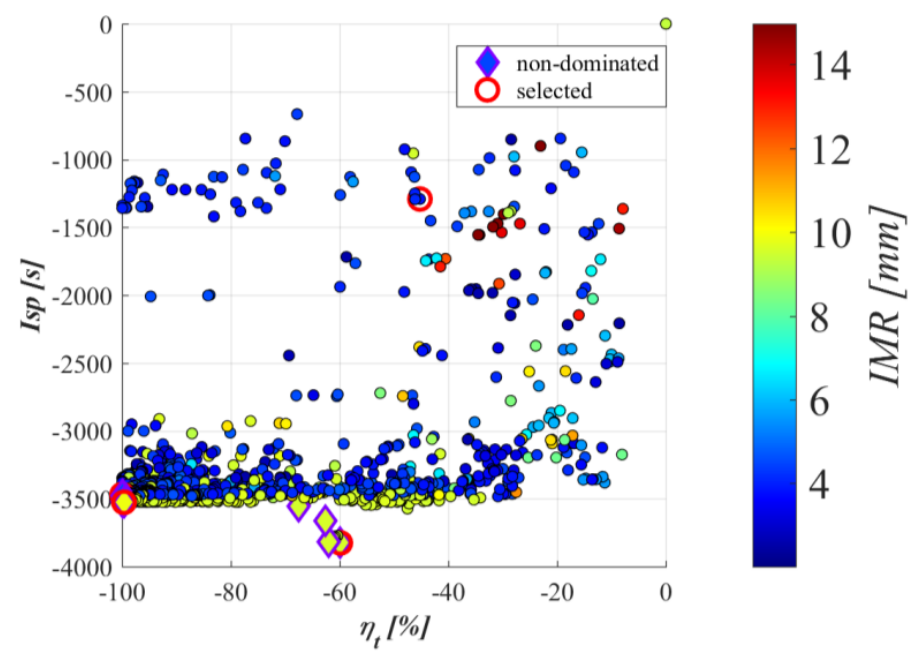

Figure 11. Inner Magnet Radii color map of the feasible solutions with respect to efficiency $\eta_{t}$ and specific impulse $I_{s p}$. 
Similar to the anode potential, the anode current, $I_{a}$, has an optimal value in the range of 2.5-3.0 A, which resulted in high $I_{s p}$ performance. Like $\Phi_{a}$, the effect of $I_{a}$ on thrust and efficiency is not as significant in comparison to specific impulses. High values for $I_{a}$ tended to offer higher efficiencies but lower thrust and $I_{s p}$. High values of $I_{a}$ have lower efficiency but comparable thrust values. Overall, an optimal range for the anode current was found within the bounds, which resulted in high performance for the objective criteria.

The effect of $m_{a}$ on the performance parameters is shown in Figure 10. Larger mass flow rates of the propellant resulted in higher thrust performance, with a fairly even distribution of $I_{s p}$ performance in regard to $m_{a}$. There are a few exceptions to this, where at mass flow rates of 20-25 (sccm) resulted in higher $I_{s p}$. Higher $m_{a}$ also resulted in higher efficiencies, while lower $m_{a}$ still produced some high-efficiency results at the expense of $I_{s p}$ performance. The non-dominated solutions resulted from high $m_{a}$ at the upper limit of the bounds, similar to the anode potential, which produced high thrust and efficiency performance for the thruster.

From Figure 11, it is evident that there are two distinct optimal value ranges for IMR which lie between 3-4 $\mathrm{mm}$ and $9-10 \mathrm{~mm}$. Values outside these ranges do not have a significant advantage for the performance objectives. The two solution ranges reflect the results of the sensitivity analysis. The IMR has the greatest influence over the strength of the magnetic field, which, in this model, is used to calculate the cusp arrival probabilities. The cusp arrival probabilities are calculated by the difference between the magnetic strength at the wall of the engine and in the far field; for our purposes, at the engine axis at the corresponding cusp location. Lower IMR values would result in higher magnetic field strengths at the wall and also subsequently in the far field. These results suggest that the optimal ratio of magnetic strengths at the cusp and in the far field exist within these two observed ranges. This is further emphasized by the magnetic field strength topologies calculated in the analysis using ANSYS Maxwell. Expectedly, OMR has similar results to IMR, with two ranges found, at approximately $13 \mathrm{~mm}$ and $25 \mathrm{~mm}$. From the sensitivity analysis, the IMR and OMR have similar influence over the performance parameters, and it will be worthwhile to consider OMR from a viewpoint of the magnet thickness because the results are somewhat ambiguous in this regard.

\section{Discussion}

A computational study has been conducted to investigate the characteristics and behavior of the HEMP-T/CFT design, aiming at the application of a downscaled thruster for microsatellite platforms. A multi-objective design optimization using surrogate-assisted evolutionary algorithms has been performed to maximize three performance measures, namely, thrust, efficiency and specific impulse, by incorporating magnetic field analysis with power distribution calculation based on the magnetic mirror condition. A global sensitivity analysis has identified the anode power, mass flow rate and magnet sizing as key design parameters and revealed sensitive, complex interactions when the design is considered holistically. The model is highly non-linear and non-convex, making it difficult to obtain a globally optimum solution. The MDO outcomes presented in Table 6 indicate evident trends of maximizing $\Phi_{a}$ and $m_{a}$ for higher performance, as well as unanticipated trends in $I_{a}$, IMR and OMR. The MDO study finds an optimal range for $I_{a}$ that does not trend towards maximizing power, and falls within the range of experimental results displayed in Table 8. The results of the geometric properties, IMR and OMR, are interesting in that they each offer two novel optimum configurations. For IMR, a local optimum is found between 3.5 and $4.0 \mathrm{~mm}$, and a global optimum is located at $9.5-10 \mathrm{~mm}$. Correspondingly, OMR has a globally optimal solution at $25 \mathrm{~mm}$ with a local optimum at $13 \mathrm{~mm}$. The results of the MDO confirm that the CFT has a geometric scalable quality which corresponds to a non-linear scale in the performance objectives. Thus, a number of novel solutions are found that reflect the observed sensitivity of geometric properties of the magnets on performance. These solutions correlate well with the limited available data of the experimental setup of Courtney et al. [7]. 
Table 8. Comparison of performance and design parameters.

\begin{tabular}{|c|c|c|c|c|c|c|}
\hline Performance Parameter & $\begin{array}{l}\text { Present } \\
\left(S_{1}\right)\end{array}$ & $\begin{array}{c}\text { Ma et al. } \\
\text { [9] }\end{array}$ & $\begin{array}{c}\text { Young et al. } \\
\text { [8] }\end{array}$ & $\begin{array}{c}\text { Courtney et al. } \\
{[7]}\end{array}$ & $\begin{array}{c}\text { Keller et al. } \\
{[3]}\end{array}$ & $\begin{array}{c}\text { Kornfeld et al. } \\
{[5]}\end{array}$ \\
\hline Efficiency $(\%)$ & 36.5 & 34.5 & 21.7 & 44.5 & 40.7 & 46 \\
\hline Anode Potential (V) & 1000 & 500 & 300 & 550 & 1100 & 1000 \\
\hline Anode Current (A) & 2.94 & 4.1 & 0.37 & 0.44 & - & 1.5 \\
\hline Mass flow rate $(\mathrm{sccm})$ & 50 & 30 & 8.2 & 8.5 & 0.48 & 15 \\
\hline Thrust $(\mathrm{mN})$ & 103 & 66 & 4.9 & 13.4 & 0.36 & 50 \\
\hline Specific Impulse (s) & 2131 & 2287 & 1239 & 1640 & 860 & 3000 \\
\hline \multicolumn{7}{|l|}{ Design Parameter } \\
\hline Inner magnet diameter (mm) & 19.82 & 40 & 17.5 & - & - & - \\
\hline Outer magnet diameter (mm) & 50.2 & 64 & 47.8 & 64 & 30 & - \\
\hline Chamber length (mm) & 50 & 96 & 39.7 & 40 & - & - \\
\hline Magnet material & 2Sm17Co & 2Sm17Co & SmCo & 3212SmCo & SmCo & SmCo \\
\hline Propellant & $\mathrm{Xe}$ & $\mathrm{Xe}$ & $\mathrm{Kr}$ & $\mathrm{Xe}$ & $\mathrm{Xe}$ & $\mathrm{Xe}$ \\
\hline Chamber wall material & $\mathrm{BN}$ & $\mathrm{BN}$ & $\mathrm{BN}$ & $\mathrm{BN}$ & $\mathrm{BN}$ & $\mathrm{BN}$ \\
\hline
\end{tabular}

The results of this MDO study on the downscaled CFT performance are able to derive some of the key relationships between the main decision variables and the performance objectives for a simplified model. An upper limit of maximum thrust was determined to be approximately $103 \mathrm{mN}$. The results of this study are compared to the available experimental data in Table 8. This is in excess of the results from Ma et al. [9], $66 \mathrm{mN}$, which was used at the baseline model for the simulation validation. It is also considerably greater than the thrust measured in experimental studies by Young et al. [8], $4.9 \mathrm{mN}$, Courtney et al. [7], $13.4 \mathrm{mN}$, Keller et al. [3], $0.48 \mathrm{mN}$, and Koch et al. [11], $50 \mathrm{mN}$. The results are also very comparable to the performances of HET and GIT. The experimental evidences did not fully explore the design space, and thus the highly non-linear relationships between the performance objectives and the decision variables were not understood adequately [5].

Table 8 compares the decision variables of the present research with those from previous studies. In comparison to the trends of the existing studies, the results are supported broadly by trends in efficiency, anode potential, and anode current. The predicted efficiency of the $S_{1}$ design is within the range of the experimental results excluding Young et al. [8]. The anode potential correlates to the setups of Reference [3,5]. The current anode is between 1.5 A [5]-4.1 A [9], where the other research utilizes much lower anode currents. The mass flow rate is much higher in this study due to in part the objectives of the research like Keller et al. [3], which aimed to minimize thrust for precision station keeping applications.

The design parameters are comparable to Young et al. [8] but represent an overall reduction in size to Ma et al. [9], and Kornfeld et al. [5] with improved performance in thrust, consequently leading to reduction in thruster weight. The comparative size of the thrusters' magnets can be optimized to produce high performance for the CFT by applying an MDO algorithm.

In comparison to the MDO study using Finite Element Method Magnetics (FEMM) analysis formerly conducted by Muffatti and Ogawa [15], an increase in all three objective functions was found. Higher $m_{a}$ and smaller IMR values indicate the overall disparity between the results. The same observations are made for higher anode potential resulting in higher $I_{s p}$ and similar performance plateaus for anode current. The geometric variables were found to have larger influence in the present MDO study coupling two-dimensional magnetostatic and electrostatic analyses than the former study with FEMM computation. The design performance was found to be highly sensitive to the anode current, $I_{a}$, and the geometric parameters of the permanent periodic magnets. Overall, an optimal current is found, along with two optimal solutions for the magnetic radii, and thus the thickness of the permanent periodic magnets. Of the selected non-dominated solutions, $S_{1}$ has been identified to be the best thruster configuration for the most beneficial performance metrics.

The present MDO study with a simplified model has provided insights into these relationships to increase the CFT performance. Further exploration of the design variables is warranted to increase the fidelity and accuracy of this model, and to include more decision variables in the analysis, with a 
greater focus on the physical thruster properties, such as chamber length, material, power to thrust ratio, and propellant.

Acknowledgments: The authors are thankful to Tapabrata Ray at the University of New South Wales, Canberra for the original $\mathrm{MDO}$ framework developed in the group.

Author Contributions: Thomas Fahey performed the numerical experiments and optimization conceived and designed by Angus Muffatti and Hideaki Ogawa in this study. Angus Muffatti and Thomas Fahey contributed to the development of the MDO process chain based on the MDO framework using surrogate-assisted evolutionary algorithms contributed by Hideaki Ogawa. Thomas Fahey and Angus Muffatti performed data analysis by applying techniques developed by Hideaki Ogawa. Thomas Fahey primarily wrote the paper with the help of Hideaki Ogawa and Angus Muffatti.

Conflicts of Interest: The authors declare no conflict of interest.

\section{References}

1. Van Noord, J. Lifetime assessment of the next ion thruster. In Proceedings of the 43rd AIAA/ASME/SAE/ ASEE Joint Propulsion Conference \& Exhibit, Cincinnati, OH, USA, 8-11 July 2007; p. 5274.

2. Goebel, D.M.; Katz, I. Fundamentals of Electric Propulsion: Ion and Hall Thrusters; John Wiley \& Sons: Hoboken, NJ, USA, 2008; Volume 1.

3. Keller, A.; Köhler, P.; Hey, F.G.; Berger, M.; Braxmaier, C.; Feili, D.; Weise, D.; Johann, U. Parametric study of HEMP-thruster downscaling to micro-newton thrust levels. IEEE Trans. Plasma Sci. 2015, 43, 45-53. [CrossRef]

4. Boyd, I.D. Simulation of Electric Propulsion Thrusters; University of Michigan: Ann Arbor, MI, USA, 2011.

5. Kornfeld, G.; Koch, N.; Harmann, H.-P. Physics and evolution of hemp-thrusters. In Proceedings of the 30th International Electric Propulsion Conference, Florence, Italy, 17-20 September 2007.

6. Koch, N.; Weis, S.; Schirra, M.; Lazurenko, A.; van Reijen, B.; Haderspeck, J.; Genovese, A.; Holtmann, P. Development, qualification and delivery status of the hempt based ion propulsion system for smallgeo. In Proceedings of the 32nd International Electric Propulsion Conference, Wiesbaden, Germany, 11-15 September 2011.

7. Courtney, D.G.; Lozano, P.; Martinez-Sanchez, M. Continued investigation of diverging cusped field thruster. In Proceedings of the 44th AIAA/ASME/SAE/ASEE Joint Propulsion Conference \& Exhibit, Hartford, CT, USA, 21-23 July 2008; Volume 4631.

8. Young, C.V.; Smith, A.W.; Cappelli, M.A. Preliminary characterization of a diverging cusped field (DCF) thruster. In Proceedings of the 31st International Electric Propulsion Conference, Ann Arbor, MI, USA, 20-24 September 2009.

9. Ma, C.; Liu, H.; Hu, Y.; Yu, D.; Chen, P.; Sun, G.; Zhao, Y. Experimental study on a variable magnet length cusped field thruster. Vacuum 2015, 115, 101-107. [CrossRef]

10. Eichmeier, J.A. Vacuum Elcectronics: Components and Devices; Springer Science \& Business Media: Berlin/Heidelberg, Germany, 2008.

11. Koch, N.; Schirra, M.; Weis, S.; Laszurenko, A.; van Reijen, B.; Haderspeck, J.; Genovese, A.; Holtmann, P.; Schneider, R.; Matyash, K. The hempt concept-A survey on theoretical considerations and experimental evidences. In Proceedings of the 32nd International Electric Propulsion Conference, Wiesbaden, Germany, 11-15 September 2011.

12. Schneider, R.; Matyash, K.; Kalentev, O.; Taccogna, F.; Koch, N.; Schirra, M. Particle-in-cell simulations for ion thrusters. Contrib. Plasma Phys. 2009, 49, 655-661. [CrossRef]

13. Genovese, A.; Lazurenko, A.; Koch, N.; Weis, S.; Schirra, M.; van Reijen, B.; Haderspeck, J.; Holtmann, P. Endurance testing of HEMPT-based ion propulsion modules for smallgeo. In Proceedings of the 32nd International Electric Propulsion Conference, Wiesbaden, Germany, 11-15 September 2011.

14. Zhurin, V.V.; Kaufman, H.R.; Robinson, R.S. Physics of closed drift thrusters. Plasma Sources Sci. Technol. 1999, 8, R5. [CrossRef]

15. Muffatti, A.; Ogawa, H. Mulit-objective design optimization of a small scale cusped field thruster for micro-satellite platforms. In Proceedings of the 16th Australian Space Research Conference, Melbourne, Australia, 26-28 September 2016; RMIT University: Melbourne, Australia, 2016. 
16. Courtney, D.G. Development and Characterization of a Diverging Cusped Field Thruster and a Lanthanum Hexaboride Hollow Cathode; Massachusetts Institute of Technology: Cambridge, MA, USA, 2008.

17. Matlock, T.S. An Exploration of Promzinent Cusped-Field Thruster Phenomena: The Hollow Conical Plume and Anode Current Bifurcation; Massachusetts Institute of Technology: Cambridge, MA, USA, 2012.

18. Löb, H.; Freisinger, J. Ionenraketen (Sammlung Vieweg); Springer: Berlin, Germany, 1967.

19. Howard, J. Introduction to Plasma Physics c17 Lecture Notes. Available online: people.physics.anu.edu.au/ jnh112/AIIM/c17/chap04.pdf (accessed on 29 July 2017).

20. Ansys Electronics Desktop Suite, version 17.2; Software Package; Ansys Inc.: Canonsburg, PA, USA, 2016.

21. Ray, T.; Smith, W. A surrogate assisted parallel multiobjective evolutionary algorithm for robust engineering design. Eng. Optim. 2006, 38, 997-1011. [CrossRef]

22. Ray, T.; Isaacs, A.; Smith, W. Multi-objective optimization using surrogate assisted evolutionary algorithm. In Introduction (G. P. Rangaiah), Multi-Objective Optimization: Techniques Applications in Chemical Engineering; World Scientific: Singapore, 2008; pp. 131-151.

23. Deb, K.; Pratap, A.; Agarwal, S.; Meyarivan, T. A fast and elitist multiobjective genetic algorithm: NSGA-II. IEEE Trans. Evol. Comput. 2002, 6, 182-197. [CrossRef]

24. Queipo, N.V.; Haftka, R.T.; Shyy, W.; Goel, T.; Vaidyanathan, R.; Tucker, P.K. Surrogate-based analysis and optimization. Prog. Aerosp. Sci. 2005, 41, 1-28. [CrossRef]

25. Lloyd, S. Least squares quantization in PCM. IEEE Trans. Inf. Theory 1982, 28, 129-137. [CrossRef]

26. Box, G.E.; Wilson, K.B. On the experimental attainment of optimum conditions. In Breakthroughs in Statistics; Springer: Berlin, Germany, 1992; pp. 270-310.

27. Buhmann, M.D. Radial Basis Functions: Theory and Implementations; Cambridge University Press: Cambridge, UK, 2003; Volume 12.

28. Rosenblatt, F. Principles of Neurodynamics: Perceptrons and the Theory of Brain Mechanisms; Cornell Aeronautical Lab Inc.: Buffalo, NY, USA, 1961.

29. Krige, D. A Statistical Approach to Some Mine Valuations and Allied Problems at the Witwatersrand; University of Witwatersrand: Johannesburg, South Africa, 1951.

30. Sobol, I. Uniformly distributed sequences with additional uniformity properties. USSR Comput. Math. Math. Phys. 1976, 16, 1332-1337. [CrossRef]

31. Saltelli, A.; Ratto, M.; Andres, T.; Campolongo, F.; Cariboni, J.; Gatelli, D.; Saisana, M.; Tarantola, S. Global Sensitivity Analysis: The Primer; John Wiley \& Sons: Hoboken, NJ, USA, 2008.

32. Koziel, S.; Yang, X.-S. Computational Optimization, Methods and Algorithms; Springer: Berlin, Germany, 2011; Volume 356.

33. Zitzler, E.; Deb, K.; Thiele, L. Comparison of multiobjective evolutionary algorithms: Empirical results. Evol. Comput. 2000, 8, 173-195. [CrossRef] [PubMed]

34. Inselberg, A. Multidimensional detective. In Proceedings of the IEEE Symposium on Information Visualization, Phoenix, AZ, USA, 21 October 1997; IEEE: Piscataway, NJ, USA, 1997; pp. 100-107. 